Statistica Sinica 13(2003), 929-953

\title{
MODELLING EXTREME-VALUE DEPENDENCE IN INTERNATIONAL STOCK MARKETS
}

\author{
Ser-Huang Poon, Michael Rockinger and Jonathan Tawn \\ University of Manchester, University of Lausanne and Lancaster University
}

\begin{abstract}
In the finance literature, cross-sectional dependence in extreme returns of risky assets is often modelled implicitly assuming an asymptotically dependent structure. If the true dependence structure is asymptotically independent then current modelling approaches will lead to an over-estimation of the risk of simultaneous extreme events. We use two simple nonparametric measures to identify and quantify the tail dependence among stock returns in five international stock markets. We show that there is strong evidence in favour of asymptotically independent models for the tail structure of stock market returns, and that most of the extremal dependence is due to heteroskedasticity in stock returns processes. Using a range of volatility filters, we find that tail index and tail dependence can be partially captured by models for heteroskedasticity. We find there is no clear reason to prefer one volatility filter over another.
\end{abstract}

Key words and phrases: Asymptotic independence, extreme value theory, Hill's estimator, risk management, tail index.

\section{Introduction}

Estimating dependence between risky asset returns is the cornerstone of portfolio theory and many other finance applications such as hedging, credit spread analysis, valuation of exotic options written on more than one asset, and risk management in general. The conventional dependence measure, Pearson correlation, though widely used is appropriate only for linear association. Since it is constructed as an average of deviations from the mean, the weight given to extreme realizations is the same as for all of the other observations in the sample. If the dependence characteristics for extreme realizations differs from all others in the sample, the conclusions drawn from the Pearson correlation could result in a financial institution risking bankruptcy. The Pearson correlation is not a good measure of dependency in cases where extreme realizations are important.

As an alternative to the traditional approach, it is possible to draw on statistical developments in extreme value theory (evt). Even though earlier applications of this field are widespread in the engineering literature, only more recently has it been brought to finance. Most applications are univariate. Jansen and de 
Vries (1991) show that the crash of 19th October 1987 may not be an isolated event. Loretan and Phillips (1994) use evt to study the existence of moments of financial returns, and Longin (1996) shows that the tails of stock market returns belong to the Fréchet class. Embrechts, Klüppelberg and Mikosch (1997) provide a summary of general evt results and comprehensive references. Diebold, Schuerman and Stroughair (1998) sketch a number of pitfalls associated with the application of evt techniques to financial data. They emphasize the role of small samples and the dependency of financial data, especially considering volatility. Kearns and Pagan (1997) show that standard errors of the tail index, that are not robust against dependency of the underlying data, may be too small. There exist some contributions, such as Harvey and Siddique (1999) and Rockinger and Jondeau (2002), where asset prices are modelled within a GARCH framework based on conditional skewness and kurtosis in addition to conditional variance. This approach may be viewed as an alternative to evt. While the number of univariate contributions increases steadily, multivariate finance applications are also beginning to appear. Longin and Solnik (2000) explore the use of multivariate extreme value methods for stock market returns, which Longin (2000) uses to demonstrate how VaR of a position can be derived. Stărică (2000) finds a high level of dependence between the extreme movements of most of the currencies in the EU. Marsh and Wagner (2000) find extremal dependence between stock returns and trading volume among equity markets. Hartmann, Straetmans and de Vries (2000) find co-crashes between stock and bond markets and some evidence of cross-border linkages for extreme events.

We draw attention to a pitfall that arises in the estimation of cross-sectional dependence among extreme returns and provide a remedy for this. The problems arise from the fact that there are two classes of extreme value dependence, asymptotic dependence and asymptotic independence, for which the characteristics of events behave quite differently as the events become more extreme. Both forms of extremal dependence permit dependence between moderately large values of each variable, but the very largest values from each variable can occur together only when the variables exhibit asymptotic dependence. To illustrate this, Figure 1 presents scatter-plots of 1,000 daily stock market returns in the US against those in Japan, and those of Germany against France. The dependence for the German-French stock market returns is persistent for both positive and negative extremes, which is indicative of the variables being asymptotically dependent. In contrast, the extremal dependence between US and Japanese stock market returns is much weaker although the largest values in each tail for one variable coincide with moderately large values of the same sign for the other variable, suggesting the variables are asymptotically independent but not exactly independent. 

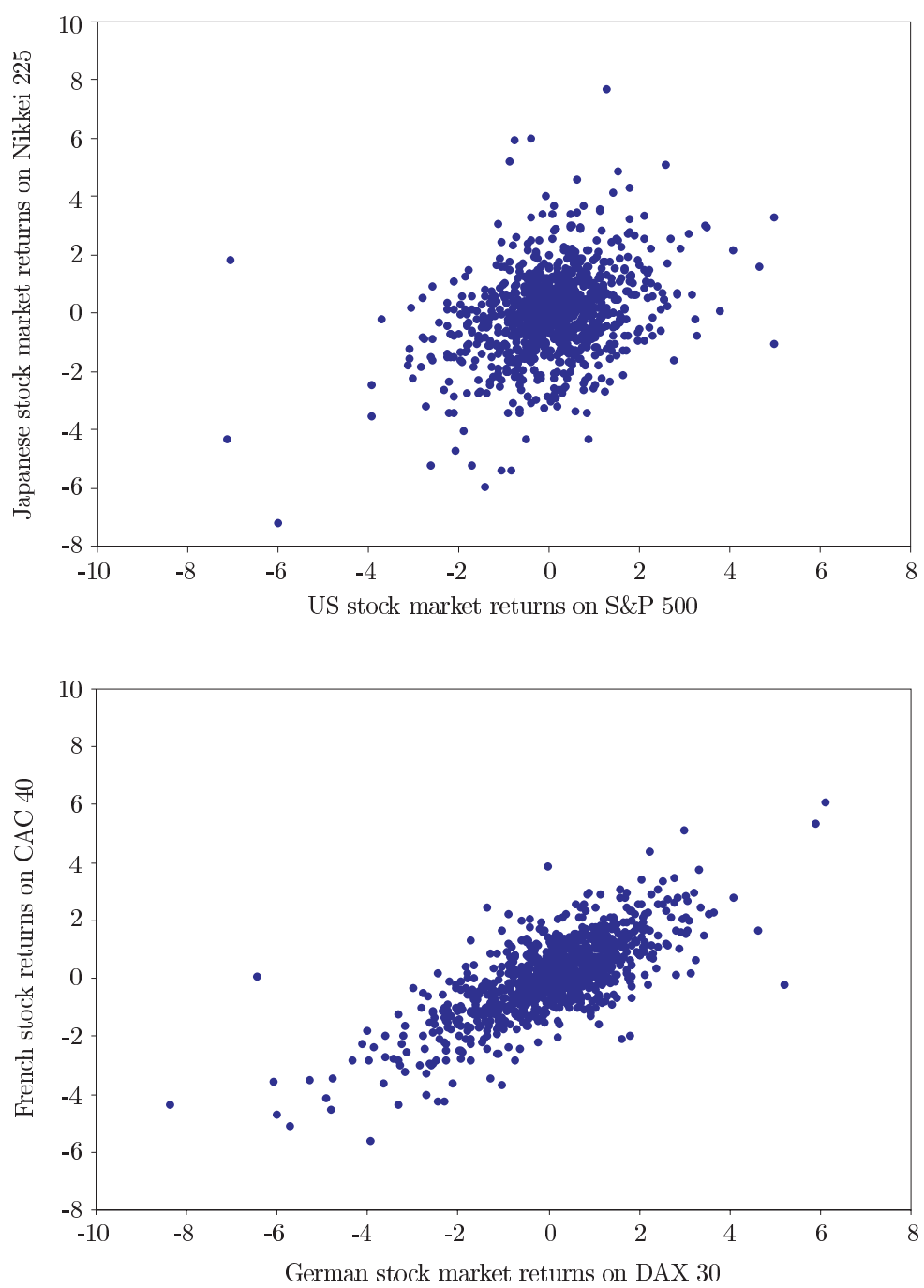

Figure 1. Scatter plot of 1,000 pairs of daily returns on selected stock market indices for the period December 19th, 1995 to May 31st, 2000.

Conventional multivariate extreme value theory has emphasized the asymptotically dependent class resulting in its wide use in all the finance applications listed above. If the series are truly asymptotically independent, such an approach will result in the over-estimation of extreme value dependence, and consequently of the financial risk. The degree of this over-estimation depends on the degree of asymptotic independence. Despite this potential for bias, the case for asymptotically independent models has so far been missing from the finance literature.

In this paper and a companion paper (Poon, Rockinger and Tawn (2003)), 
we use techniques developed by Ledford and Tawn (1997) and Coles, Heffernan and Tawn (1999) to distinguish between asymptotically dependent and asymptotically independent variables, and to quantify the degree of dependence for the appropriate dependence class. Here we document the effect of heteroskedasticity on the tail index and tail dependence among international stock market returns, and we examine the effect of volatility filtering on tail estimates. Finally we demonstrate, using a nonparametric approach, how portfolio risk may be assessed. We refer the readers to our companion paper for the parametric setting. In contrast with the parametric approach, the non-parametric approach is less precise, but it does not require any assumption on the functional form of the joint tail distribution. We view the two approaches as complementary.

Using daily returns on five stock indices (viz. S\&P, FTSE, DAX, CAC and Nikkei) over a 31.5-year period from December 26th, 1968 to May 31st, 2000, we find left-tail dependence to be usually stronger than right-tail dependence. In addition, we demonstrate that many of these stock index returns do not exhibit asymptotic dependence, suggesting that much of the extreme value dependence reported in previous studies is likely to be over-estimated. With the use of volatility filters, we find that most of the extreme value dependence is caused by changing stock market volatility, but detect little difference in the results produced by the three volatility filters. Using the US and Japanese stock return pair as an example, we show how an erroneous assumption of asymptotic tail dependence could lead to portfolio risk being overestimated.

The remaining sections are organized as follows: Section 2 briefly describes univariate extreme value theory and recent developments in the measurement of dependence in multivariate extreme values. Section 3 describes the empirical analyses, which include a description of the data sources and a report of empirical findings. Section 4 provides a brief discussion on how the concepts underpinning the measures for extreme value dependence can be made into useful portfolio management tools. Section 5 offers conclusions.

\section{Extreme Value Theory and Extremal Dependence}

To assess the financial risk of a portfolio using Extreme Value Theory, the complete joint distribution of the various assets during periods of great turmoil needs to be estimated. This involves estimating the marginal distributions and the dependence structure. We focus on dependence estimation in the bivariate context, though the ideas and techniques extend naturally to higher dimensions. In the first subsection below, we briefly describe univariate extreme value methods as they are used both to determine the marginal distributions and to provide 
the inference techniques for the dependence measures described in the second subsection.

\subsection{Univariate methods}

There is a long history, and a large associated literature, on probability characterizations and statistical models for univariate extremes. The numerous approaches by which extreme values may be statistically modelled come in two forms: methods for maxima over fixed intervals, and methods for exceedances over high thresholds. We outline the fundamental aspects of each method. Further details can be found in Embrechts, Klüppelberg and Mikosch (1997) and Reiss and Thomas (1997).

The limit theory for the maximum of a sample of $n$ independent and identically distributed random variables is based on a location-scale normalization of the maximum so that its distribution is non-degenerate as $n \rightarrow \infty$. Provided a non-degenerate limit can be achieved, the limit distribution of the maximum must be the Gumbel, the Fréchet or the negative Weibull distributions. The generalized extreme value distribution (GEV) is a unifying model that encompasses these three types of extreme value distributions. It has three parameters, $\mu, \sigma$ and $\xi$, denoting the location, scale and shape parameters respectively. The shape parameter, $\xi$, also called the tail index, determines the three extreme value types. Specifically, when $\xi$ takes negative values, positive values or 0 , the GEV distribution is the negative Weibull, the Fréchet or the Gumbel distribution, respectively. For example, if the original variables follow a normal distribution then a Gumbel distribution will result for the maximum. Similarly, the Fréchet (negative Weibull) distributions arise as the distribution of the maximum for variables with heavier (lighter) tails than the normal. There is now a growing consensus that many financial series have heavy tails (see for example, Loretan and Phillips (1994)), so the Fréchet distribution is to be expected.

When observations on all exceedances of a high threshold are available, then using only fixed interval maximum values is inefficient as this may exclude large observations from the analysis. The appropriate limit theory in this context is one based on a point process result of Pickands (1971), which has been advocated for statistical modelling by Smith (1989). The limit result suggests modelling exceedances of a high threshold by a non-homogeneous Poisson process. A consequence of this model is that the excess values over the threshold follow the generalized Pareto distribution (GPD) and that maximum values are modelled by the generalized extreme value distribution, both distributions having a common shape parameter $\xi$. The GPD model, advocated by Pickands (1975) and Davison and Smith (1990), provides a flexible family of tail behaviours, with $\xi=0$ corresponding to the exponential distribution. A key modelling aspect 
with threshold methods is the selection of the threshold. A number of diagnostic techniques exist for threshold selection, including a bootstrap method which produces an optimal value that minimizes the empirical mean square errors of the tail index (Danielsson and de Vries (1997)). The critical aspect of threshold selection is that inferences should be insensitive to increases in threshold above the optimal level.

For the subsequent dependence measures we introduce a special example of threshold modelling linked to the generalized Pareto distribution for the case where $\xi>0$, i.e., Fréchet tail. In this case the tail of the variable $Z$ above a high threshold $u$ can be approximated as

$$
\operatorname{Pr}(Z>z) \sim \frac{\mathcal{L}(z)}{z^{1 / \xi}} \text { for } z>u
$$

where $\mathcal{L}(z)$ is a slowly varying function of $z$ (see Embrechts, Klüppelberg and Mikosch (1997, p.325). Treating the slowly varying function as a constant for all $z>u$, say $\mathcal{L}(z)=c$, and under the assumption of independent observations, the maximum likelihood estimators for $\xi$, known as Hill's estimator (Hill (1975)), and $c$ are

$$
\begin{aligned}
& \hat{\xi}=\frac{1}{n_{u}} \sum_{j=1}^{n_{u}} \log \left(\frac{z_{(j)}}{u}\right), \\
& \hat{c}=\frac{n_{u}}{n} u^{1 / \hat{\xi}},
\end{aligned}
$$

where $z_{(1)}, \ldots, z_{\left(n_{u}\right)}$ are the $n_{u}$ observations of variable $Z$ that exceed $u$.

The above discussion applies to independent variables. When the variables are dependent, the statistical approaches for analyzing maxima are unchanged as the limit distribution of the maximum is also a generalized extreme value distribution. Unlike the maximum over intervals method, temporal dependence due to the use of threshold method adds some complications. Ignoring the dependence and applying the methods as if the data were independent will lead to unbiased estimators but with standard errors that are too small (Kearns and Pagan (1997)). Several approaches may be used to overcome this problem: declustering of the exceedances of the threshold to produce approximately independent data (see Davison and Smith (1990)), or adjusting the standard error within a maximum likelihood framework (see Coles and Walshaw (1994)).

In this paper, the standard errors of the parameters of the marginal and joint tails are evaluated under the assumption of independence over time. To assess the validity of this assumption we use the reciprocal of the extremal index, the standard measure of clustering of extreme values in univariate stationary time series, to estimate the average size of clusters of extreme values (see Smith 
and Weissman (1994)). Small average cluster sizes are found, typically 1.2 observations for the raw series and 1.1 for the filtered and squared filtered series. Consequently, the effect of ignoring temporal dependence on the standard errors used in this paper is likely to be minimal.

\subsection{Measuring extreme value dependence}

Much effort has been made to extend univariate extreme value theory for applications in a multivariate context. In almost all multivariate studies, it is helpful to remove the influence of marginal aspects first by transforming the original variables to a common marginal distribution. After such a transformation, differences in distributions are purely due to dependence aspects. Hence our dependence measures, unlike correlation, are no longer influenced by the form of the marginal distribution. For a further discussion of this issue, see Embrechts, McNeil and Strautman (2002). In this spirit, we transform the bivariate returns $(X, Y)$ to unit Fréchet marginals $(S, T)$ using the transformation

$$
S=-1 / \log F_{X}(X) \text { and } T=-1 / \log F_{Y}(Y),
$$

where $F_{X}$ and $F_{Y}$ are the respective marginal distribution functions for $X$ and $Y$. Consequently, $\operatorname{Pr}(S>s)=\operatorname{Pr}(T>s) \sim s^{-1}$ as $s \rightarrow \infty$, and $(S, T)$ possess the same dependence structure as $(X, Y)$. In practice, the values of $F_{X}$ and $F_{Y}$ that are used in the transformation (4) are obtained using the empirical distribution functions of the separate variables.

\subsubsection{The conventional approach}

To understand extremal dependence, one must first appreciate that the form and degree of such dependence determine the chance of obtaining large values of both variables. As $S$ and $T$ are on a common scale, events of the form $\{S>s\}$ and $\{T>s\}$, for large values of $s$, correspond to equally extreme events for each variable. As all such probabilities will tend to zero as $s \rightarrow \infty$ it is natural to consider conditional probabilities of one variable given that the other is extreme. Specifically, consider the behaviour of $\operatorname{Pr}(T>s \mid S>s)$ for large $s$. If $(S, T)$ are perfectly dependent then $\operatorname{Pr}(T>s \mid S>s)=1$. In contrast, if $(S, T)$ are exactly independent then $\operatorname{Pr}(T>s \mid S>s)=\operatorname{Pr}(T>s)$, which tends to 0 as $s \rightarrow \infty$. If

$$
\chi=\lim _{s \rightarrow \infty} \operatorname{Pr}(T>s \mid S>s),
$$

$0 \leq \chi \leq 1$, we say that variables are asymptotically dependent if $\chi>0$ and asymptotically independent if $\chi=0$. Clearly $\chi$ measures the degree of dependence that persists to the limit. An example of a non-trivial asymptotically dependent 
joint distribution is the logistic model in the bivariate extreme value family, see Tawn (1988) and Longin and Solnik (2000), which for unit Fréchet margins has

$$
\operatorname{Pr}(S \leq s, T \leq t)=\exp \left\{-\left(s^{-1 / \alpha}+t^{-1 / \alpha}\right)^{\alpha}\right\}
$$

with $0<\alpha \leq 1$. When $\alpha=1$, the variables are exactly independent and $\chi=0$. When $\alpha<1, \chi=2-2^{\alpha}$ and the variables are asymptotically dependent to a degree depending on $\alpha$. Generally, when $\chi=0$ the two random variables are not necessarily exactly independent. For example, if the dependence structure is that of a bivariate normal random variable with any value for the correlation coefficient less than one, then $\chi=0$ (Sibuya (1960)).

When exact independence is rejected, traditional multivariate extreme value methods assume $\operatorname{Pr}(T>s \mid S>s)=\chi>0$ for all large $s$. See for example de Haan (1985), de Haan and de Ronde (1998) and Coles and Tawn (1991, 1994). If the true distribution of the variables is asymptotically independent, the use of the traditional multivariate extreme value methods will over-estimate $\operatorname{Pr}(S>s, T>$ $s$ ) and all other probabilities of joint extreme events since $\operatorname{Pr}(T>s \mid S>s) \rightarrow 0$ as $s \rightarrow \infty$. The degree of bias will depend on the difference between the estimated $\chi$ and the true value of $\operatorname{Pr}(T>s \mid S>s)$, which is determined by the value of $s$ and the rate at which $\operatorname{Pr}(T>s \mid S>s) \rightarrow 0$ as $s \rightarrow \infty$.

\subsubsection{An alternative measure of dependence}

More recently, Ledford and Tawn (1996, 1997), Bruun and Tawn (1998), Bortot and Tawn (1998) have provided a range of extremal dependence models, derived from a different form of multivariate limit theory, that describe dependence but have $\chi=0$. Although the random variables are asymptotically independent in this case, different degrees of dependence are attainable at finite levels of $s$. Based on these studies, Coles, Heffernan and Tawn (1999) suggest that

$$
\bar{\chi}=\lim _{s \rightarrow \infty} \frac{2 \log \operatorname{Pr}(S>s)}{\log \operatorname{Pr}(S>s, T>s)}-1,
$$

$-1<\bar{\chi} \leq 1$, is an appropriate measure of asymptotic independence as it gives the rate that $\operatorname{Pr}(T>s \mid S>s) \rightarrow 0$. Values of $\bar{\chi}>0, \bar{\chi}=0$ and $\bar{\chi}<0$ loosely correspond, respectively, to when $(S, T)$ are positively associated in the extremes, exactly independent, and negatively associated. For the bivariate normal dependence structure $\bar{\chi}$ is the correlation coefficient. For other examples see Heffernan (2000).

The pair of dependence measures $(\chi, \bar{\chi})$ together provide all the necessary information to characterise the form and degree of extremal dependence. For asymptotically dependent variables $\bar{\chi}=1$ with the degree of dependence given by $\chi>0$. For asymptotically independent variables $\chi=0$ with the degree of 
dependence given by $\bar{\chi}$. It is important to test if $\bar{\chi}=1$ first before drawing conclusions about asymptotic dependence based on estimates of $\chi$.

\section{3. $\bar{\chi}$ and $\chi$ : Estimation and statistical inference}

For estimating $\bar{\chi}$ and $\chi$, weak assumptions are required (the first three of which are essentially the same as those required when estimating the univariate tail behaviour by extreme value models): (i) the joint distribution $(S, T)$ has a joint tail behaviour that is bivariate regularly varying, satisfying the conditions of Ledford and Tawn (1998)-counter examples to this form are given by Schlather (2001); (ii) both $\bar{\chi}$ and $\chi$ are limit properties, so it is necessary to assume that the sample characteristics of the empirical joint distribution, above some selected threshold, reflect the limiting behaviour; (iii) the series has sufficient independence over time for the sample characteristics to converge to the population characteristics $\bar{\chi}$ and $\chi$; (iv) the marginal variables can be transformed to identically distributed Fréchet variables.

With the above assumptions, we use results in Ledford and Tawn (1996, $1997,1998)$ to estimate $\bar{\chi}$ and $\chi$, where it was established that, under weak conditions,

$$
\operatorname{Pr}(S>s, T>s) \sim \mathcal{L}(s) s^{-1 / \eta} \quad \text { as } \quad s \rightarrow \infty,
$$

$0<\eta \leq 1$ is a constant and $\mathcal{L}(s)$ is a slowly varying function. From this representation it follows that

$$
\bar{\chi}=2 \eta-1
$$

and that if $\bar{\chi}=1$, corresponding to $\eta=1$, then $\chi=\lim _{s \rightarrow \infty} \mathcal{L}(s)$. Thus estimating $\eta$ and $\lim _{s \rightarrow \infty} \mathcal{L}(s)$ provide the basis for estimating $\chi$ and $\bar{\chi}$. There is the possibility that $\eta=1$ and $\mathcal{L}(s) \rightarrow 0$ as $s \rightarrow \infty$ leading to asymptotic independence. This boundary case cannot be identified from data as the slowly varying function cannot be identified other than as a constant, and misspecification of the dependence structure in this situation is unlikely to be important. Thus, we focus on inference for $\eta$ and $\lim _{s \rightarrow \infty} \mathcal{L}(s)$, treating the slowly varying function as constant over some threshold $u$, i.e. $\mathcal{L}(s)=d$ for $s>u$.

Inference follows using univariate extreme value techniques by noting that if $Z=\min (S, T)$,

$$
\begin{aligned}
\operatorname{Pr}(Z>z)= & \operatorname{Pr}\{\min (S, T)>z\}=\operatorname{Pr}(S>z, T>z)=\mathcal{L}(z) z^{-1 / \eta}=d z^{-1 / \eta} \\
& \text { for } z>u,
\end{aligned}
$$

for some high threshold $u$. From this representation and the univariate tail form (11), it can be seen that $\eta$ is the tail index of the univariate variable $Z$, and so can be easily estimated using the Hill estimator from (2), truncated to the interval $(0,1]$, and that $d$ is the associated scale parameter which can be estimated as at (3). 
The following development is based on the assumption of independent observations on $Z$ to obtain our estimator for $\bar{\chi}$ :

$$
\begin{gathered}
\hat{\bar{\chi}}=\frac{2}{n_{u}}\left(\sum_{j=1}^{n_{u}} \log \left(\frac{z_{(j)}}{u}\right)\right)-1, \\
\operatorname{Var}(\hat{\bar{\chi}})=(\hat{\bar{\chi}}+1)^{2} / n_{u},
\end{gathered}
$$

with the notation as in equations (2) and (3), and the asymptotically normality of $\widehat{\bar{\chi}}$ ensured by results in Smith (1987). If $\widehat{\bar{\chi}}$ is significantly less than 1 (i.e., if $\hat{\bar{\chi}}+$ $1.96 \sqrt{\operatorname{Var}(\hat{\bar{\chi}})}<1)$ then we infer the variables to be asymptotically independent and take $\chi=0$. Only if there is no significant evidence to reject $\bar{\chi}=1$ do we estimate $\chi$, which we do under the assumption that $\bar{\chi}=\eta=1$. Using the maximum likelihood estimator given by (3) and under the constraint $\hat{\bar{\chi}}=1$, our estimator of $\chi$ is

$$
\begin{gathered}
\widehat{\chi}=\frac{u n_{u}}{n}, \\
\operatorname{Var}(\widehat{\chi})=\frac{u^{2} n_{u}\left(n-n_{u}\right)}{n^{3}} .
\end{gathered}
$$

Furthermore, we can assess whether the variables have a joint tail which decays with the same form as for exact independence by testing if $\hat{\bar{\chi}}$ is significantly different from 0 .

\section{Empirical Analyses}

Our data consists of closing stock index levels of S\&P 500 from the US, FTSE 100 from the UK, DAX 30 from Germany, CAC 40 from France and Nikkei 225 from Japan. Our sample period spans from December 26th, 1968 to May 31st, 2000, giving rise to 8,200 daily return observations for each series. Three of the indices (viz. S\&P, FTSE and CAC) were created by grafting two returns series from the same country. For example, the UK returns are represented by the FT All Shares returns before 1st January 1980 and FTSE returns after that date. Daily index returns are generated by taking first differences of the logarithmic indices. Although some of the returns series do not include the dividend distribution, dividends do not generate extreme movements so this is not a problem for our analysis.

It has been widely documented elsewhere that the US market has, by far, the greatest influence on all the other stock markets (see, for example, Martens and Poon (2001)). The US market is also the latest to close on a calendar day among the five stock markets in our sample. This means that any extreme movements in the US stock market are likely to impact on the other stock markets on the 
following day. As a result, contemporaneous day observations will underestimate the dependence between US and other stock markets. To overcome this problem, we adopt the synchronization procedures in Martens and Poon (2001), where the dependence measure is calculated between the non-US returns and a weighted sum of today's and yesterday's US returns. The weights are derived by optimizing this dependence measure. However, we find that the results reported in the following sections are not sensitive to the synchronization procedure or the weighting scheme adopted. This is because extreme returns tend to cluster and extremes have equal chances of originating from the US and outside the US.

Zero returns due to holidays may cause estimators to be biased. It turns out that when the focus is on the tails of the distribution, there is no difference whether one removes the zero returns or not. Nevertheless, for the analyses in the following sections, we have removed all zero returns in all univariate models. In the bivariate case, both observations of a particular day are removed if at least one of the two observations is a zero return.

\subsection{Descriptive statistics}

Table 1 presents some summary statistics for the five stock index returns. The mean, variance, skewness and excess kurtosis statistics, computed using Richardson and Smith (1993) generalized method of moment procedures, are robustness against heteroskedasticity. The average for the five mean returns is $0.033 \%$ (or $8.5 \%$ per annum) excluding dividends. All five returns series have a negative skewness, which means that extreme negative returns are a dominant feature for all five indices. Excess kurtosis is significantly greater than zero for all series. The excess kurtosis for US stock returns is particularly high, suggesting Fréchet type tails for US stock returns distribution.

The Engle(1) statistic is large and highly significant, indicating strong heteroskedasticity for all five stock returns series. The Portmanteau test for stock returns autocorrelation among the first five lags, $Q(5)$, is reported in Table 1. Due to the presence of strong heteroskedasticity, the standard errors and the associated $p$-values are computed following Hansen (1982) and Nicholls and Pagan (1983). The null hypothesis of no autocorrelation is rejected for UK, Germany and France, and is marginally rejected for the US returns. This suggests returns series are autocorrelated, which may affect the degree of dependence across series. For this reason, we fit an autoregressive filter to all stock returns.

Table 2 presents descriptive statistics for returns that are filtered for autocorrelation and those that are filtered for heteroskedasticity in addition. Three volatility filters were used to remove heteroskedasticity: AGARCH is an asymmetric version of GARCH; ADC is the Asymmetric Dynamic Covariance model in Kroner and $\mathrm{Ng}$ (1998), which is a multivariate GARCH model; SV is the stochastic volatility model in Kim, Shephard and Chib (1998). Detail specifications of 
the volatility filters are presented in the Appendix. ADC filtered residuals were derived by fitting a bivariate ADC model to US and UK returns. The filtered residuals for German, French and Japanese stock returns come, respectively, from fitting each of the return series with the US returns (as the second series) in the bivariate ADC model. We find descriptive statistics of filtered residuals to be insensitive to the choice of the second stock returns series used in the ADC model.

Table 1. Descriptive statistics for daily stock index returns over the period December 26th, 1968 to May 31st, 2000.

\begin{tabular}{|lccccc|}
\hline & US & UK & Germany & France & Japan \\
\hline Mean & 0.032 & 0.035 & 0.031 & 0.039 & 0.028 \\
Std. Err. & $(0.011)$ & $(0.012)$ & $(0.012)$ & $(0.012)$ & $(0.012)$ \\
Std Deviation & 0.953 & 1.042 & 1.082 & 1.055 & 1.092 \\
Std. Err. & $(0.036)$ & $(0.019)$ & $(0.020)$ & $(0.020)$ & $(0.025)$ \\
Skewness & -1.791 & -0.313 & -0.555 & -0.610 & -0.164 \\
Std. Err. & $(1.498)$ & $(0.313)$ & $(0.297)$ & $(0.318)$ & $(0.469)$ \\
Excess Kurtosis & 45.110 & 9.413 & 9.668 & 10.173 & 15.168 \\
Std. Err. & $(33.709)$ & $(2.995)$ & $(2.871)$ & $(3.346)$ & $(5.230)$ \\
Engle(1) & 108.383 & 1973.137 & 313.459 & 79.355 & 325.174 \\
$p$-value & $(0.000)$ & $(0.000)$ & $(0.000)$ & $(0.000)$ & $(0.000)$ \\
$Q(5)$ & 10.908 & 30.536 & 12.935 & 59.595 & 7.435 \\
$p$-value & $(0.053)$ & $(0.000)$ & $(0.024)$ & $(0.000)$ & $(0.190)$ \\
\hline
\end{tabular}

Notes: The stock market indices are S\&P 500 (for the US), FTSE 100 (for the UK), DAX 30 (for Germany), CAC 40 (for France) and Nikkei 225 (for Japan). The mean, variance, skewness and excess kurtosis statistics, computed using Richardson and Smith (1993) generalized method of moment procedures, are robust against heteroskedasticity. The Engle(1) statistic is calculated as $T \times R^{2}$, where $T$ is the sample size and $R^{2}$ is the coefficient of determination for the regression of squared centered returns on a constant and lagged squared returns of order 1. $Q(5)$ is the Portmanteau test for stock returns autocorrelation among the first five lags. The standard error and $p$-value of the Portmanteau test statistic, computed following Hansen (1982) and Nicholls and Pagan (1983), are robust against heteroskedasticity.

We note from Table 2 that the AR filtered returns have retained much of the characteristics of the raw returns except that skewness and $Q(5)$, the Portmanteau test for autocorrelation, are no longer significantly different from zero. The AR filtered residuals continue to have thick tails and heteroskedasticity. Excess kurtosis and heteroskedasticity were successfully removed by all three heteroskedasticity filters. Hence, returns that are filtered for heteroskedasticity are now much closer to being i.i.d. The SV residuals, in particular, are approximately Gaussian as the skewness and excess kurtosis are the cloest to zero. 
Table 2. Descriptive statistics for AR filtered returns series over the period December 26th, 1968 to May 31st, 2000.

\begin{tabular}{|c|c|c|c|c|c|}
\hline & US & UK & Germany & France & Japan \\
\hline \multicolumn{6}{|c|}{ Returns not filtered for heteroskedasticity } \\
\hline Skewness & -1.707 & -0.257 & -0.556 & -0.595 & -0.313 \\
\hline Std. Err. & $(-63.117)$ & $(-9.516)$ & $(-20.534)$ & $(-21.989)$ & $(-11.565)$ \\
\hline Excess Kurtosis & 43.043 & 8.512 & 9.729 & 10.761 & 14.153 \\
\hline Std. Err. & $(795.569)$ & $(157.330)$ & $(179.827)$ & $(198.888)$ & $(261.594)$ \\
\hline Engle(1) & 191.374 & 2096.846 & 368.428 & 147.171 & 399.572 \\
\hline$p$-value & $(0.000)$ & $(0.000)$ & $(0.000)$ & $(0.000)$ & $(0.000)$ \\
\hline$Q(5)$ & 0.004 & 0.002 & 0.018 & 0.003 & 0.002 \\
\hline$p$-value & $(1.000)$ & $(1.000)$ & $(1.000)$ & $(1.000)$ & $(1.000)$ \\
\hline \multicolumn{6}{|c|}{ AGARCH filtered residuals } \\
\hline Skewness & -0.385 & -0.474 & -0.718 & -0.497 & -0.468 \\
\hline Std. Err. & $(-14.230)$ & $(-17.527)$ & $(-26.525)$ & $(-18.365)$ & $(-17.311)$ \\
\hline Excess Kurtosis & 4.116 & 5.091 & 9.670 & 8.243 & 8.411 \\
\hline Std. Err. & $(76.049)$ & $(94.068)$ & $(178.680)$ & $(152.306)$ & $(155.405)$ \\
\hline Engle(1) & 3.641 & 5.874 & 0.046 & 0.017 & 0.927 \\
\hline$p$-value & $(0.602)$ & $(0.319)$ & $(1.000)$ & $(1.000)$ & $(0.968)$ \\
\hline$Q(5)$ & 4.057 & 2.150 & 5.217 & 7.947 & 11.590 \\
\hline$p$-value & $(0.945)$ & $(0.995)$ & $(0.876)$ & $(0.634)$ & $(0.313)$ \\
\hline \multicolumn{6}{|c|}{ ADC filtered residuals } \\
\hline Skewness & -0.330 & -0.300 & -0.665 & -0.468 & -0.316 \\
\hline Std. Err. & $(-12.094)$ & $(-11.000)$ & $(24.425)$ & $(-17.192)$ & $(-11.641)$ \\
\hline Excess Kurtosis & 3.483 & 2.105 & 8.282 & 7.567 & 6.285 \\
\hline Std. Err. & $(63.904)$ & $(38.612)$ & $(152.163)$ & $(138.937)$ & $(115.622)$ \\
\hline Engle(1) & 0.763 & 3.067 & 0.005 & 0.007 & 0.081 \\
\hline$p$-value & $(0.979)$ & $(0.690)$ & $(1.000)$ & $(1.000)$ & $(1.000)$ \\
\hline$Q(5)$ & 5.409 & 1.989 & 5.072 & 7.583 & 11.580 \\
\hline$p$-value & $(0.862)$ & $(0.996)$ & $(0.886)$ & $(0.669)$ & $(0.314)$ \\
\hline \multicolumn{6}{|c|}{ SV filtered residuals } \\
\hline Skewness & -0.043 & -0.074 & -0.067 & 0.013 & -0.101 \\
\hline Std. Err. & $(-1.569)$ & $(-2.718)$ & $(-2.456)$ & $(0.481)$ & $(-3.720)$ \\
\hline Excess Kurtosis & -0.120 & -0.389 & -0.415 & -0.439 & -0.487 \\
\hline Std. Err. & $(-2.208)$ & $(-7.185)$ & $(-7.664)$ & $(-8.116)$ & $(-9.004)$ \\
\hline Engle(1) & 1.274 & 1.613 & 0.202 & 0.737 & 0.083 \\
\hline$p$-value & $(0.938)$ & $(0.900)$ & $(0.999)$ & $(0.981)$ & $(1.000)$ \\
\hline$Q(5)$ & 4.583 & 2.202 & 6.774 & 14.339 & 15.099 \\
\hline$p$-value & $(0.917)$ & $(0.995)$ & $(0.747)$ & $(0.158)$ & $(0.129)$ \\
\hline
\end{tabular}

Notes: The stock market indices are S\&P 500 (for the US), FTSE 100 (for the UK), DAX 30 (for Germany), CAC 40 (for France) and Nikkei 225 (for Japan). All stock market returns are filtered for autocorrelation using an AR filter with five autoregressive terms. The mean, variance, skewness and excess kurtosis, computed using Richardson and Smith (1993) generalized method of moment procedures, are robust against heteroskedasticity. The Engle(1) statistic is calculated as $T \times R^{2}$, where $T$ is the sample size and $R^{2}$ is the coefficient of determination for the regression of squared centered returns on a constant and lagged squared returns of order 1. $Q(5)$ is the Portmanteau test for stock returns autocorrelation among the first five lags. The standard error and $p$-value of the Portmanteau test statistic, computed following Hansen (1982) and Nicholls and Pagan (1983), are robust against heteroskedasticity. 


\subsection{Univariate tail indices}

In this study, all tail indices and tail dependence structure are separately estimated for left and right tails. Table 3 presents tail indices estimates for returns filtered for autocorrelation and those that are filtered for heteroskedasticity in addition. Following the Danielsson and de Vries (1997) method for optimal threshold selection, the threshold estimated for our sample of 8,200 observations typically contains $2 \%$ of the data in the tail. Estimation results, details not reported here, suggest that the tail index estimate is quite stable within a small variation of the threshold selected using this method. The stability of the tail index with respect to the choice of small variations of the threshold confirms the findings in Lux (2001).

Table 3. Tail index estimates for daily stock market returns over the period December 26th, 1968 to May 31st, 2000.

\begin{tabular}{|c|c|c|c|c|c|}
\hline & $\mathrm{US}$ & UK & Germany & France & Japan \\
\hline \multicolumn{6}{|c|}{ Returns not filtered for heteroskedasticity } \\
\hline \multirow[t]{2}{*}{ Left tail } & 0.3179 & 0.2907 & 0.2999 & 0.3368 & 0.3165 \\
\hline & $(0.0171)$ & $(0.0221)$ & $(0.0242)$ & $(0.0220)$ & $(0.0270)$ \\
\hline \multirow[t]{2}{*}{ Right tail } & 0.2731 & 0.3300 & 0.3067 & 0.2768 & 0.3562 \\
\hline & $(0.0229)$ & $(0.0328)$ & $(0.0216)$ & $(0.0203)$ & $(0.0288)$ \\
\hline \multicolumn{6}{|c|}{ AGARCH filtered residuals } \\
\hline \multirow[t]{2}{*}{ Left tail } & 0.2455 & 0.2350 & 0.2394 & 0.2702 & 0.2735 \\
\hline & $(0.0172)$ & $(0.0156)$ & $(0.0174)$ & $(0.0186)$ & $(0.0195)$ \\
\hline \multirow[t]{2}{*}{ Right tail } & 0.1895 & 0.2018 & 0.2137 & 0.2205 & 0.2980 \\
\hline & $(0.0155)$ & $(0.0146)$ & $(0.0148)$ & $(0.0167)$ & $(0.0195)$ \\
\hline \multicolumn{6}{|c|}{ ADC filtered residuals } \\
\hline \multirow[t]{2}{*}{ Left tail } & 0.2363 & 0.2328 & 0.2527 & 0.2666 & 0.2707 \\
\hline & $(0.0168)$ & $(0.0152)$ & $(0.0167)$ & $(0.0171)$ & $(0.0205)$ \\
\hline \multirow[t]{2}{*}{ Right tail } & 0.1925 & 0.1933 & 0.2176 & 0.2132 & 0.2936 \\
\hline & $(0.0169)$ & $(0.0157)$ & $(0.0143)$ & $(0.0166)$ & $(0.0202)$ \\
\hline \multicolumn{6}{|c|}{ SV filtered residuals } \\
\hline \multirow[t]{2}{*}{ Left tail } & 0.1346 & 0.1466 & 0.1425 & 0.0926 & 0.0853 \\
\hline & $(0.0111)$ & $(0.0117)$ & $(0.0105)$ & $(0.0076)$ & $(0.0069)$ \\
\hline \multirow[t]{2}{*}{ Right tail } & 0.1172 & 0.1351 & 0.1205 & 0.0919 & 0.0892 \\
\hline & $(0.0097)$ & $(0.0110)$ & $(0.0102)$ & $(0.0075)$ & $(0.0073)$ \\
\hline
\end{tabular}

Notes: The stock market indices are S\&P 500 (for the US), FTSE 100 (for the UK), DAX 30 (for Germany), CAC 40 (for France) and Nikkei 225 (for Japan). All stock market returns are filtered for autocorrelation using an AR filter with five autoregressive terms. The tail index is estimated based on Hill estimator using a bootstrap method for selecting optimal threshold following Danielsson and de Vries (1997). Numbers presented in parentheses are standard errors.

From Table 3 we note that tail index estimates are significantly reduced when stock returns are filtered for heteroskedasticity, and the reduction is the 
most dramatic when the SV filter is used. Note that the tail index of a Gaussian variable is equal to zero, whereas all the tail indices reported in Table 3 are significantly greater than zero even after the returns are filtered for autocorrelation and heteroskedasticity. There is no significant evidence that the left and right tail indices are different from one another for each of the series we consider, independent of the type of volatility filter used.

\subsection{Extremal dependence}

Campbell and Hentschel (1993) suggest that news arrival creates volatility, which in turn leads to an increase in the risk premium and a fall in stock prices. The fall in stock price is greater if the news is bad, and less severe if the news is good. We argue further that bad news tends to affect several stock markets at the same time creating a co-movement among their volatility and a stronger dependence among international stock markets during crises. The empirical findings in the following sections generally support this conjecture.

\subsubsection{Whole period analysis}

Table 4 reports tail dependency $\bar{\chi}$ estimates for the left and right tails of selected pairs of stock index returns that are filtered for autocorrelation and the corresponding heteroskedasticity filtered counterparts. Note that $-1<\bar{\chi} \leq 1$ from (7), but our estimation based on the Hill's estimator in (2) is not constrained to satisfy the upper bound. Table 4 shows that all $\bar{\chi}$ estimates are greater than zero but the tail dependence of heteroskedasticity filtered returns is substantially reduced. This is true for both right and left tail dependence with little difference between the three volatility filters. All (except two) of the $\bar{\chi}$ estimates for the left tail are larger than those for the right tail. Hence, we may conclude that it is much more likely for the five international stock markets to exhibit joint crashes than to have simultaneous upswings. This corresponds to findings reported in Ang and Chen (2000), Longin and Solnik (2000) and Martens and Poon (2001).

Inspection of the tail indices and associated standard errors indicates that the only case where asymptotic dependence cannot be rejected (i.e., $\bar{\chi}=1$ ) is between German and French returns when they are both not filtered for heteroskedasticity. All filtered returns are asymptotically independent (i.e., $\bar{\chi}<1$ ). From the interpretation of $\bar{\chi}$ in Section 2.2, this implies that there is significant dependence between large values of the paired series but that the very largest values do not occur concurrently. Since most of the pairs are asymptotically independent, multivariate extreme value models that assume asymptotic dependency among stock market returns are likely to have overestimated portfolio joint risk. In Section 4 we assess how important this error is by obtaining an approximation to the bias in a portfolio risk assessment that is incurred by falsely assuming asymptotic dependence. 
Table 4. Measures of tail independence, $\bar{\chi}$, for selected daily stock market return pairs.

\begin{tabular}{|c|c|c|c|c|c|c|c|c|}
\hline \multirow[b]{4}{*}{ US-UK } & \multicolumn{4}{|c|}{$\begin{array}{l}\text { Returns not filtered for } \\
\text { heteroskedasticity }\end{array}$} & \multicolumn{4}{|c|}{ AGARCH filtered residuals } \\
\hline & \multicolumn{2}{|c|}{ Left tail } & \multicolumn{2}{|c|}{ Right tail } & \multicolumn{2}{|c|}{ Left tail } & \multicolumn{2}{|c|}{ Right tail } \\
\hline & $\bar{\chi}$ & s.e. & $\bar{\chi}$ & s.e. & $\bar{\chi}$ & s.e. & $\bar{\chi}$ & s.e. \\
\hline & 0.592 & 0.092 & 0.605 & 0.114 & 0.364 & 0.054 & 0.317 & 0.066 \\
\hline US-GER & 0.661 & 0.075 & 0.456 & 0.057 & 0.528 & 0.064 & 0.248 & 0.057 \\
\hline US-FRA & 0.507 & 0.060 & 0.358 & 0.070 & 0.472 & 0.070 & 0.296 & 0.051 \\
\hline US-JAP & 0.479 & 0.059 & 0.510 & 0.106 & 0.395 & 0.055 & 0.262 & 0.098 \\
\hline UK-GER & 0.854 & 0.115 & 0.676 & 0.116 & 0.495 & 0.057 & 0.290 & 0.076 \\
\hline UK-FRA & 0.752 & 0.109 & 0.511 & 0.063 & 0.630 & 0.093 & 0.351 & 0.055 \\
\hline \multirow[t]{3}{*}{ GER-FRA } & 1.191 & 0.155 & 0.817 & 0.115 & 0.614 & 0.080 & 0.353 & 0.054 \\
\hline & \multicolumn{4}{|c|}{ ADC filtered residuals } & \multicolumn{4}{|c|}{ SV filtered residuals } \\
\hline & \multicolumn{2}{|c|}{ Left tail } & \multicolumn{2}{|c|}{ Right tail } & \multicolumn{2}{|c|}{ Left tail } & \multicolumn{2}{|c|}{ Right tail } \\
\hline & $\bar{\chi}$ & s.e. & $\bar{\chi}$ & s.e. & $\bar{\chi}$ & s.e. & $\bar{\chi}$ & s.e. \\
\hline US-UK & 0.392 & 0.051 & 0.279 & 0.051 & 0.386 & 0.052 & 0.283 & 0.051 \\
\hline US-GER & 0.471 & 0.068 & 0.268 & 0.056 & 0.532 & 0.065 & 0.264 & 0.056 \\
\hline US-FRA & 0.397 & 0.059 & 0.279 & 0.048 & 0.349 & 0.081 & 0.287 & 0.052 \\
\hline US-JAP & 0.374 & 0.068 & 0.302 & 0.056 & 0.367 & 0.066 & 0.326 & 0.053 \\
\hline UK-GER & 0.538 & 0.071 & 0.278 & 0.066 & 0.446 & 0.058 & 0.285 & 0.061 \\
\hline UK-FRA & 0.608 & 0.089 & 0.330 & 0.055 & 0.659 & 0.121 & 0.362 & 0.054 \\
\hline GER-FRA & 0.558 & 0.062 & 0.324 & 0.061 & 0.552 & 0.069 & 0.336 & 0.067 \\
\hline
\end{tabular}

Notes: The stock market indices are S\&P 500 (for the US), FTSE 100 (for the UK), DAX 30 (for Germany), CAC 40 (for France) and Nikkei 225 (for Japan). All stock market returns are filtered for autocorrelation using an AR filter with five autoregressive terms. $\bar{\chi}$ is computed based on the tail index of Frechet transformed margins of daily co-exceedances of stock market returns pair. Standard errors (s.e.) are computed as $(\hat{\bar{\chi}}+1) / \sqrt{n_{u}}$, where $n_{u}$ is the number of observations exceeding the threshold $u$.

\subsubsection{Subperiod analysis}

In this section, we focus on the stability of dependence structure across time. To keep the analysis and reporting of results manageable, we omit all filtering exercises in this subsection. Table 5 presents unconstrained $\bar{\chi}$ estimates for selected pairs of unfiltered stock index returns, across three non-overlapping subperiods. Each subperiod is over ten years, with the world market crash taking place in subperiod 2 and the integration of the European Union evolving throughout subperiod 3. The percentage of observations that exceed the thresholds is stable across the three subperiods for each returns pair. For example, the percentages of observations falling into the left tail of German-French unfiltered returns distribution in subperiods 1,2 and 3 are $6 \%, 6 \%$ and $5 \%$ respectively. The corresponding figures for US-UK unfiltered returns are 3\%, 5\% and $6 \%$ for the right tail. 
Table 5. Two measures of tail dependence, $\bar{\chi}$ and $\chi$, for selected daily stock market return pairs over three sub-periods.

\begin{tabular}{|c|c|c|c|c|c|c|c|c|c|}
\hline \multicolumn{10}{|c|}{ Subperiod 1: 27 December 1968 - 19 June 1979 (2,733 observations) } \\
\hline & & \multicolumn{2}{|c|}{ Left tail } & \multicolumn{2}{|c|}{ Right tail } & \multicolumn{2}{|c|}{ Left tail } & \multicolumn{2}{|c|}{ Right tail } \\
\hline & $\rho$ & $\bar{\chi}$ & s.e. & $\bar{\chi}$ & s.e. & $\bar{\chi}$ & s.e. & $\bar{\chi}$ & s.e. \\
\hline US-UK & 0.220 & 0.472 & 0.118 & 0.601 & 0.170 & \multirow{7}{*}{0.239} & \multirow{7}{*}{0.024} & & \\
\hline US-GER & 0.211 & 0.820 & 0.185 & 0.355 & 0.098 & & & & \\
\hline US-FRA & 0.261 & 0.381 & 0.109 & 0.683 & 0.238 & & & 0.173 & 0.025 \\
\hline US-JAP & 0.116 & 0.333 & 0.104 & 0.368 & 0.095 & & & & \\
\hline UK-GER & 0.102 & 0.332 & 0.097 & 0.299 & 0.089 & & & & \\
\hline UK-FRA & 141 & 0.502 & 0.125 & 0.344 & 0.092 & & & & \\
\hline GER-FRA & 0.163 & 0.438 & 0.111 & 0.183 & 0.127 & & & & \\
\hline \multicolumn{10}{|c|}{ Subperiod 2: 20 June 1979 - 8 December 1989 (2,733 observations) } \\
\hline & & \multicolumn{2}{|c|}{ Left tail } & \multicolumn{2}{|c|}{ Right tail } & \multicolumn{2}{|c|}{ Left tail } & \multicolumn{2}{|c|}{ Right tail } \\
\hline & $\rho$ & $\bar{\chi}$ & s.e. & $\bar{\chi}$ & s.e. & $\bar{\chi}$ & s.e. & $\bar{\chi}$ & s.e. \\
\hline US-UK & 0.347 & 0.800 & 0.243 & 0.765 & 0.156 & 0.195 & 0.026 & 0.257 & 0.022 \\
\hline US-GER & 0.319 & 0.688 & 0.116 & 0.442 & 0.106 & & & & \\
\hline US-FRA & 0.257 & 0.568 & 0.107 & 0.272 & 0.081 & & & & \\
\hline US-JAP & 0.407 & 0.626 & 0.111 & 0.764 & 0.193 & & & 0.238 & 0.026 \\
\hline ER & 0.345 & 0.658 & 0.114 & 0.591 & 0.173 & & & & \\
\hline UK & 0.287 & 0.845 & 0.234 & 0.449 & 0.102 & 0.222 & 0.028 & & \\
\hline GER-FRA & 0.358 & 0.809 & 0.140 & 0.518 & 0.104 & 0.309 & 0.023 & & \\
\hline \multicolumn{10}{|c|}{ Subperiod 3: 11 December 1989 - 31 May 2000 (2,733 observations) } \\
\hline & & \multicolumn{2}{|c|}{ Left tail } & \multicolumn{2}{|c|}{ Right tail } & \multicolumn{2}{|c|}{ Left tail } & \multicolumn{2}{|c|}{ Right tail } \\
\hline & $\rho$ & $\bar{\chi}$ & s.e. & $\bar{\chi}$ & s.e. & $\bar{\chi}$ & s.e. & $\bar{\chi}$ & s.e. \\
\hline US- & 0.311 & 0.724 & 0.177 & 0.462 & 0.119 & 0.275 & 0.028 & & \\
\hline ER & 0.361 & 0.593 & 0.110 & 0.452 & 0.099 & & & & \\
\hline & 0.275 & 0.575 & 0.109 & 0.345 & 0.123 & & & & \\
\hline US-JAP & 0.264 & 0.482 & 0.118 & 0.493 & 0.114 & & & & \\
\hline UK-GER & 0.570 & 1.043 & 0.166 & 0.850 & 0.142 & 0.421 & 0.033 & 0.361 & 0.0 \\
\hline UK-FRA & 0.670 & 0.824 & 0.167 & 0.711 & 0.136 & & & & \\
\hline GER-FRA & 0.664 & 1.023 & 0.177 & 0.913 & 0.156 & 0.476 & 0.041 & 0.413 & 0.033 \\
\hline
\end{tabular}

Notes: The stock market indices are S\&P 500 (for the US), FTSE 100 (for the UK), DAX 30 (for Germany), CAC 40 (for France) and Nikkei 225 (for Japan). $\rho$ is the correlation coefficient calculated using all observations in the subperiod. $\bar{\chi}$ and $\chi$ are computed based on tail index estimation on Frechet transformed margins of daily co-exceedances of stock market returns pair. For cases where asymptotic dependence cannot be rejected (i.e. $\bar{\chi}=1), \chi$ is computed assuming that $\hat{\bar{\chi}}$ is equal to 1 .

There is evidence of complex non-stationarity in the extremal dependence with a general increase over time in the $\bar{\chi}$ estimates for the left tail. The same pattern is found for the right tail amongst the European markets. Table 5 also presents the Pearson correlation coefficients. All the correlation coefficients are significantly positive and appear to have increased through time, especially 
among the European countries.

Estimates of $\chi$ for pairs where asymptotic dependence cannot be rejected are also reported in Table 5. There are twelve of these; five for the right tails and seven for the left tails. In most of these cases, they are pertaining to the left tail dependence within the European markets and in the last decade. This provides a considerable strengthening to the understanding provided by Pearson correlation coefficients.

\section{Implications of Asymptotic Independence for Portfolio Risk Assess- ment}

In this section, we focus on estimating portfolio risk. The emphasis here is to show that portfolio risk will be over-estimated when based on the assumption of asymptotic dependence when the returns are asymptotically independent. Here, we follow the Ledford and Tawn (1997) approach for handling asymptotic independence to develop a method for providing bounds on the portfolio risk. These are relatively tight if the variables are asymptotically dependent, but for asymptotically independent variables the bounds differ, with the lower bound being the more likely to provide a better approximation in many cases. In a companion paper, Poon, Rockinger and Tawn (2003), we adopt parametric models for the joint tail distributions. Here we adopt a nonparametric approach. Bounds of the portfolio returns distribution are inferred directly from the $\chi$ and $\bar{\chi}$ estimates produced from the previous section without additional distributional dependence assumptions.

To illustrate the estimation of bounds on portfolio risk we focus on the bivariate case. Define $(X, Y)$ to be two returns. In a portfolio risk management context, one would seek a convex combination of these returns, i.e., $a X+(1-a) Y$ for $0<a<1$, such that the probability of the combination exceeding a high threshold, $k$, is minimized over $a$. The key stage of this process is the evaluation of the probability for a given $a$. This is the aspect we focus on, estimating $\operatorname{Pr}\{a X+(1-a) Y>k\}$ for fixed $a$ and $k$, with $k$ large. For any $(x, y)$, where $y=(k-a x) /(1-a)$, we obtain

$$
\operatorname{Pr}(X>x, Y>y) \leq \operatorname{Pr}(a X+(1-a) Y>k) \leq 1-\operatorname{Pr}(X<x, Y<y) .
$$

Generally, these bounds will be uninformative, however the point $(x, y)$ can be selected to minimize the errors for each bound simultaneously. The appropriate $(x, y)$ point is the one with the largest joint density, which generally occurs when $x$ and $y$ are at equal marginal quantile values, i.e., we seek $(x, y)$ with $y=$ $(k-a x) /(1-a)$ and

$$
\operatorname{Pr}(X>x)=\operatorname{Pr}(Y>y)
$$


Define this point by $\left(x_{0}, y_{0}\right)$. To evaluate $\left(x_{0}, y_{0}\right)$ we need a model for the marginal distribution tail form. From Section 2.1 the univariate tail model (11) is appropriate for finance data, with different parameters $c_{X}, \xi_{X}$ and $c_{Y}, \xi_{Y}$ for each margin. Thus, (16) corresponds to $y_{0}=\left(c_{Y} / c_{X}\right)^{\xi_{Y}} x_{0}^{\xi_{Y} / \xi_{X}}$. It follows that $\operatorname{Pr}\left(X>x_{0}, Y>y_{0}\right)=\operatorname{Pr}\left(S>s_{0}, T>s_{0}\right)$ and $1-\operatorname{Pr}(X<x, Y<y)=$ $2 \operatorname{Pr}\left(X>x_{0}\right)-\operatorname{Pr}\left(S>s_{0}, T>s_{0}\right)$, where $(S, T)$ have unit Fréchet marginal distributions, and $s_{0} \approx 1 / \operatorname{Pr}\left(X>x_{0}\right)=x_{0}^{\xi_{X}} / c_{x}=y_{0}^{\xi_{Y}} / c_{Y}$. Then (15) becomes $\operatorname{Pr}\left(X>x_{0}\right) \operatorname{Pr}\left(T>s_{0} \mid S>s_{0}\right) \leq \operatorname{Pr}(a X+(1-a) Y>k) \leq \operatorname{Pr}(X>$ $\left.x_{0}\right)\left\{2-\operatorname{Pr}\left(T>s_{0} \mid S>s_{0}\right)\right\}$, so from expressions (8) and (10),

$$
d s_{0}^{-(1-\bar{\chi}) /(1+\bar{\chi})} \leq \operatorname{Pr}(a X+(1-a) Y>k) / \operatorname{Pr}\left(X>x_{0}\right) \leq 2-d s_{0}^{-(1-\bar{\chi}) /(1+\bar{\chi})} .
$$

Using estimators of the marginal tail parameters and estimators of the extremal dependence structure characteristics, $d$ and $\bar{\chi}$, these bounds can be evaluated. Together with the estimate of $\operatorname{Pr}\left(X>x_{0}\right)$, these bounds provide the required bounds on $\operatorname{Pr}(a X+(1-a) Y>k)$. If the variables are asymptotically dependent, so $\bar{\chi}=1$ and $d=\chi$, then as $k \rightarrow \infty$ the bounds on $\operatorname{Pr}(a X+(1-a) Y>$ $k) / \operatorname{Pr}\left(X>x_{0}\right)$ converge to $\chi$ and $2-\chi$, so are very tight. For asymptotically independent variables these limits for the bounds are 0 and 2, respectively. However the rate of approach to these limits depends on the degree of asymptotic independence, $\bar{\chi}$. Typically, for finite $k$, the lower bound in (17) provides a much closer approximation to the truth than the upper bound.

Figure 2 presents the upper and lower bounds of the portfolio risk, $\operatorname{Pr}(a X+$ $(1-a) Y>k)$, calculated based on (17) for two country pairs, viz., US vs. Japan and Germany vs. France, using left tail parameter estimates for the most recent subperiod unfiltered returns. The solid lines in the first graph are generated by "wrongly" assuming that US and Japanese stock market returns are asymptotically dependent. Since tail dependence of stock returns that are not filtered for heteroskedasticity exhibit an intertemporal instability, the use of subperiord estimates is more appropriate. Also, to avoid the complication caused by changing volatility, we use $\chi$ and $\bar{\chi}$ estimated from unfiltered returns. Current research on the estimation of portfolio risk under the influence of heteroskedasticity is still ongoing.

To provide an example, we assume in each portfolio that the weights for the first and second assets are 0.25 and 0.75 respectively. The daily percentage loss, $k$, is selected such that it is always greater than the individual univariate extreme value thresholds obtained through bootstrap estimation, as mentioned in Section 2.1. As one would expect when the daily percentage loss $k$ increases, the logarithmic expected waiting time between such losses in Figure 2 increases, and the band between the upper and lower bounds widens. We observed previously from Table 5 that asymptotic dependence is rejected for the US-Japanese returns 
US and Japanese Stock Markets

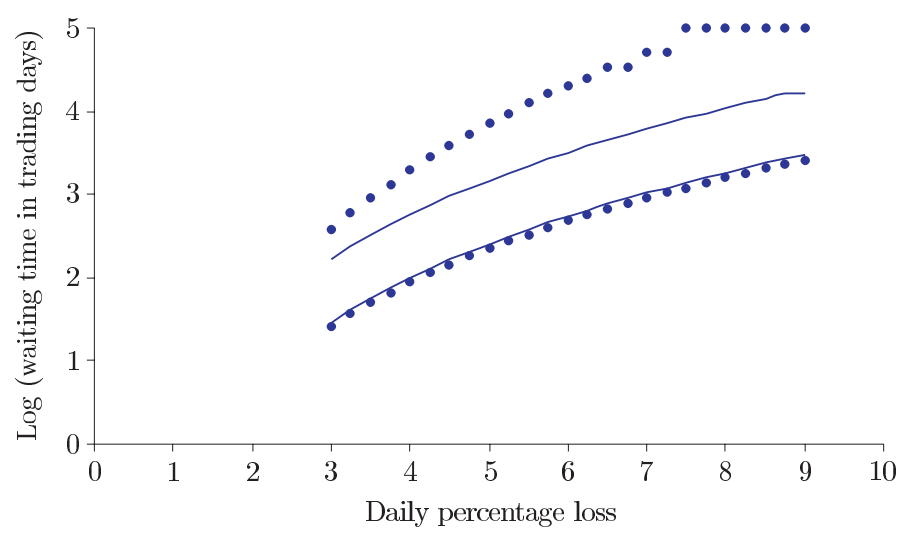

German and French Stock Markets

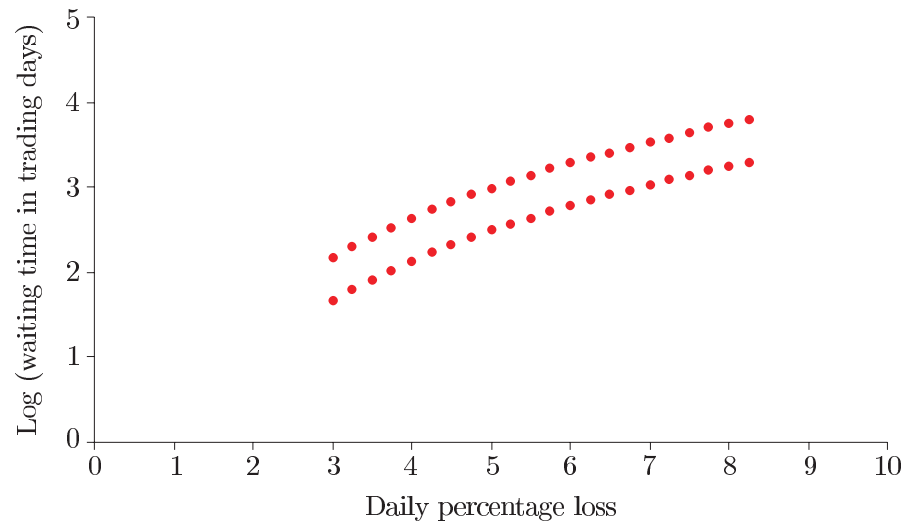

Figure 2. Bounds on portfolio risk estimated for subperiod 3 from 11 December 1989 to 31 May 2000.

Note: Portfolio weights for first and second assets are 0.25 and 0.75 respectively. The soild lines in first graph are generated by "wrongly" assuming that US and Japanese stock market returns are asymptotically dependent.

pair, but cannot be rejected for the German-French returns pair. This corresponds to the wider band between upper and lower bounds of the US-Japanese portfolio compared with that of the German-French portfolio. If we translate the probability of portfolio risk into a waiting time between threshold exceedances, the probability of the US-Japanese portfolio having a one-day loss equal to or exceeding $3 \%$ ranges between 1.2 and 17.2 months. The estimated waiting time for the equivalent loss for the German-French portfolio ranges between only 2 and 7 months. On the other hand, if we had erroneously concluded that US 
and Japanese returns were asymptotic dependent, the waiting time would have been 1.3 and 7.8 months. In term of one-day loss estimates, the one-day loss will be overstated as $3.614 \%$ and $8.25 \%$ for fixed level of lower bound probability, whereas the true one-day loss should be $3 \%$ and $5.5 \%$, respectively. For finite level of one-day loss, the lower bound provides a much closer approximation to the truth than the upper bound. These overstatements of one-day loss will exert a wrong influence on the portfolio composition in an environment where risk management is an important aspect of the internal control system.

\section{Conclusion}

In this paper, we use two non-parametric measures for extreme value dependency to characterise tail dependence of returns in five international stock markets and demonstrate how portfolio tail risk may be assessed. These new tools have allowed us to document, for the first time, the widespread asymptotic independence among stock market returns, a phenomenon that has so far been overlooked in the finance literature. The omission of asymptotic independence models has led to over-estimation, possibly substantial, of portfolio risk.

Other empirical findings include a confirmation that extreme value dependence is much stronger in bear markets than in bull markets, and that much of the extreme value dependency is due to correlated conditional volatility. We find tail indices and tail dependence to be substantially reduced after the stock returns are filtered for heteroskedasticity. Three volatility filters were tested but we found there is little to choose between them. This suggests that in modelling tail dependence, it is acceptable to use a univariate GARCH for modelling and controlling the effect of heteroskedasticity.

In general, the correlation between volatilities has increased over time to produce asymptotically dependent stock markets within Europe and strong, but still asymptotically independent stock markets between Europe (UK, Germany and France), North America (US) and Asia (Japan).

An accurate measurement of asset-returns behaviour during periods of extreme movements is useful in many finance applications. With the new dependency measures, the dynamics of conditional correlation can be better understood. Further work could investigate the hedging efficiency gain and option pricing improvement with a model for conditional correlation that captures the characteristics observed here. The modelling of portfolio joint tail distribution would require detailed calibration of individual tail distributions and asset extreme value dependency. Ongoing research in this area should provide better tools for portfolio management and risk diversification. 


\section{Acknowledgements}

Ser-Huang Poon started this project when she was at Lancaster University. She wants to thank the Unversity Research Committee for financial support. Rockinger, who is also at FAME and CEPR, acknowledges financial support from TMR (grant on Financial market's efficiency) and the Swiss National Science Foundation through NCCR (Financial Valuation and Risk Management). We would like to thank Ruey Tsay and seminar participants at Lancaster University, University of Lausanne, Quantitative Finance Conference at Academia Sinica (Taipei 2001) and European Finance Association Meetings (Berling 2002) for helpful comments. We are grateful to the referee and the editor for their comments and suggestions.

\section{Appendix}

This appendix describes the filters used in Section 3. There are two types of filters; one used to removed autocorrelation in stock returns and a second used to remove stock returns heteroskedasticity. Three volatility models were used to filter out heteroskedasticity, viz. AGARCH (Asymmetric GARCH), ADC (Asymmetric Dynamic Covariance) and SV (Stochastic Volatility). ADC is a bivariate GARCH filter, whereas AGARCH and SV are both univariate filters.

\section{A.1. AR filter for autocorrelation in returns}

The filter used to remove autoregressive dependency is an $\operatorname{AR}(5)$ model: $R_{t}=\mu+\sum_{j=1}^{5} \alpha_{j} R_{t-j}+\epsilon_{t}$. Stock returns, $R_{t}$, were replaced by the residuals $\epsilon_{t}$ in the estimation of tail index and tail dependence.

\section{A.2. AGARCH filter for heteroskedasticity}

The first filter for heteroskedasticity we use is an asymmetric version of the GARCH(1,1) model, see Zakoïan (1994), which is based on the model that the return $R_{t}$ at time $t$ follows a normal distribution with mean $\omega$ and standard deviation $\sqrt{h_{t}}$, where $R_{t}=\omega+\sqrt{h_{t}} Z_{t}$ and $h_{t}=\alpha_{0}+\alpha^{+} Z_{t-1}^{2} h_{t-1} D_{Z_{t-1} \geq 0}+$ $\alpha^{-} Z_{t-1}^{2} h_{t-1} D_{Z_{t-1}<0}+\beta h_{t-1}$. Here $\alpha_{0}, \alpha^{+}, \alpha^{-}$and $\beta$ are parameters, and $D_{E}$ is the indicator function that event $E$ occurs. We refer to this filter as AGARCH. Using estimated values of these parameters the filtered series of $Z_{t}$ values is derived to replace stock returns in the estimation of tail index and tail dependence.

\section{A.2. SV filter for heteroskedasticity}

A second, more general form of volatility filter, is the stochastic volatility model presented by Kim, Shephard and Chib (1998): $R_{t}=\beta e^{h_{t} / 2} Z_{t}, h_{t}=\mu+$ 
$\phi\left(h_{t-1}-\mu\right)+\sigma_{\eta} \eta_{t}, h_{1} \sim \mathcal{N}\left(\mu, \sigma^{2} /\left(1-\phi^{2}\right)\right)$, where $h_{t}$ is interpreted as the log volatility at time $t$. The innovations $Z_{t}$ and $\eta_{t}$ are uncorrelated standard normal white noise shocks. For identifiability reasons, either $\beta$ must be set to one or $\mu$ to zero. These parameters play the role of scaling variables.

Whereas all the other estimations of this paper are done under GAUSS, the estimation of the stochastic volatility model was achieved using Ox. We downloaded Ox from the website of Jurgen Doornik (http://www.nuff.ox.ac.uk/users/ doonik) and obtained the code used in the Kim, Shephard and Chib (1998) from the web-site http://www.nuff.ox.ac.uk/users/sheppard/ox). After experimenting with the various estimation methods, we settled for the Gibbs sampling algorithm presented in Kim, Shephard and Chib (1998). We refer to the stochastic volatility model as the SV filter.

\section{A.2. ADC bivariate GARCH filter}

There have been studies documenting volatility spillover from one country to the other. To take care of this type of phenomenon, we also consider a generalized multivariate GARCH model of Kroner and Ng (1998), which also captures asymmetric dynamic covariances (ADC). To describe this model, we use the notations $R_{i t}$ for time $t$ rate of return of asset $i=1, \ldots, N$. The expected return, given all information at time $t-1$ is $\mu_{i t}$, which will either be a constant or a constant plus $\operatorname{AR}(5), Z_{i t}$ is the unexpected return of asset $i, Z=\left[Z_{1 t}, \ldots, Z_{N t}\right]^{\prime}$, $h_{i t}$ is the conditional variance of $R_{i t}$ given all information at time $t-1, h_{i j t}$ is the conditional covariance between $R_{i t}$ and $R_{j t}$. Let $H_{t}$ be the conditional covariance matrix $\left(H_{t}=\left[h_{i j t}\right]_{i, j}\right)$.

Introducing $\eta_{i t}=\max \left(0,-Z_{i t}\right)$ and $\eta_{t}=\left[\eta_{1 t}, \ldots, \eta_{N t}\right]^{\prime}$ the ADC model that we consider here is $h_{i j t}=w_{i j}+b_{i}^{\prime} H_{t-1} b_{j}+a_{i}^{\prime} Z_{t-1} Z_{t-1}^{\prime} a_{j}+g_{i}^{\prime} \eta_{t-1} \eta_{t-1} g_{j}, \forall i, j$, where $a_{i}, b_{i}$, and $g_{i}, i=1, \ldots, N$, are parameters. The $w_{i j}$ are scalars. The ADC model may be viewed as a multivariate extension of the previous AGARCH model.

\section{References}

Ang, A. and Chen, J. (2000). Asymmetric correlations of equity portfolio. Working paper, Columbia University.

Bortot, P. and Tawn, J. A. (1998). Models for the extremes of Markov chains. Biometrika 85, 851-867.

Bruun, J. T. and Tawn, J. A. (1998). Comparison of approaches for estimating the probability of coastal flooding. Appl. Statist. 47, 405-423.

Campbell, J. Y. and Hentschel, L. (1992). No news is good news: An asymmetric model of changing volatility in stock returns. J. Finan. Econom. 31, 281-318.

Coles, S. G. and Tawn, J. A. (1991). Modelling extreme multivariate events. J. Roy. Statist. Soc. Ser. B 53, 377-392. 
Coles, S. G. and Tawn, J. A. (1994). Statistical methods for multivariate extremes: An application to structural design (with discussion). Appl. Statist. 43, 1-48.

Coles, S. G., Heffernan, J. and Tawn, J. A. (1999). Dependence measures for extreme value analyses. Extremes 3, 5-38.

Coles, S. G. and Walshaw, D. (1994). Directional modelling of extreme wind speeds. Appl. Statist. 43, 139-157.

Davison, A. C. and Smith, R. L. (1990). Models for exceedances over high thresholds (with discussion). J. Roy. Statist. Soc. Ser. B 52, 393-442.

Danielsson, J. and de Vries, C. G. (1997). Tail index and quantile estimation with very high frequency data. J. Empirical Finance 4, 241-257.

Diebold, F. X., Schuermann, T. and Stroughair, J. D. (1998). Pitfalls and opportunities in the use of extreme value theory in risk management. In Computational Finance (Edited by P. Refenes). Kluwer Acadmic Press, Boston.

de Haan, L. (1985). Extremes in high dimensions: the model and some statistics. In Proc. 45th Sess. Internat. Statist. Inst., paper 26.3. International Statistical Institute, the Hague.

de Haan, L. and de Ronde, J. (1998). Sea and wind: multivariate extremes at work. Extremes 1, 7-45.

Embrechts, P., Klüppelberg, C. and Mikosch, T. (1997). Modeling Extremal Events. Springer Verlag, New York.

Embrechts, P., McNeil, A. and Strautman, D. (2002). Correlation and dependency in risk management: properties and pitfalls. In Risk Management: Value at Risk and Beyond (Edited by M. A. H. Dempster). Cambridge University Press.

Hansen, L. P. (1982). Large sample properties of generalized method of moments estimators. Econometrica 50, 1029-1054.

Hartmann, P., Straetmans, S. and de Vries, C. G. (2000). Asset market linkages in crisis periods. Working paper, Erasmus University, Rotterdam.

Harvey, C. R. and Siddique, A. (1999). Autoregressive conditional skewness. J. Finan. Quant. Anal. 34, 465-487.

Heffernan, J. E. (2000). Directory of coefficients of tail dependence. Extremes 3, 279-290.

Hill, B. M. (1975). A simple general approach to inference about the tail of a distribution. Ann. Statist. 3, 1163-1173.

Jansen, D. W. and De Vries, C. G. (1991). On the frequency of large stock returns: putting booms and busts into perspectives. Rev. Econom. Statist. 73, 18-24.

Kearns, P. and Pagan, A. (1997). Estimating the density tail index for financial time series. Rev. Econom. Statist. 79, 171-175.

Kim, S., Shephard, N. and Chib, S. (1998). Stochastic volatility, likelihood inference, and comparison with ARCH models. Rev. Econom. Stud. 65, 361-393.

Kroner, K. F. and Ng, V. K. (1998). Modeling asymmetric comovements of asset returns. Rev. Finan. Stud. 11, 817-844.

Ledford, A. W. and Tawn, J. A. (1996). Statistics for near independence in multivariate extreme values. Biometrika $\mathbf{8 3}, \mathbf{1 6 9 - 1 8 7 .}$

Ledford, A. W. and Tawn, J. A. (1997). Modeling dependence within joint tail regions. J. Roy. Statist. Soc. Ser. B 49, 475-499.

Ledford, A. W. and Tawn, J. A. (1998). Concomitant tail behaviour for extremes. Adv. Appl. Probab. 30, 197-215.

Longin, F. M. (1996). The Asymptotic Distribution of Extreme Stock Market Returns. J. Bus. 63, 383-408.

Longin, F. M. (2000). From value at risk to stress testing: The extreme value approach. $J$. Banking Finance 24, 1097-1130. 
Longin, F. M. and Solnik, B. (2000). Extreme correlation of international equity markets. J. Finance 56, 649-676.

Loretan, M. and Phillips, P. C. B. (1994). Testing the covariance stationarity of heavy-tailed time series. J. Empirical Finance 1, 211-248.

Lux, T. (2001). The limiting extremal behaviour of speculative returns: An analysis of intradaily data from the frankfurt stock exchange. Appl. Finan. Econom. 11, 299-315.

Marsh, T. A. and Wagner, N. (2000). Return-volume dependence and extremes in international markets. Working paper, Haas School of Business, University of California, Berkeley.

Martens, M. and Poon, S. (2001). Returns synchronization and daily correlation dynamics between international stock markets. J. Banking Finance 25, 1805-1827.

Nicholls, D. F. and Pagan, A. R. (1983). Heteroscedasticity in models with lagged dependent variables. Econometrica 51, 1233-1242.

Pickands, J. (1971). The two-dimensional Poisson process and extremal processes. J. Appl. Probab. 8, 745-756.

Pickands, J. (1975). Statistical inference using extreme order statistics. Ann. Statist. 3, 119-131.

Poon, S., Rockinger, M. and Tawn, J. A. (2003). Extreme-value dependence in financial markets: diagnostics, models and financial implications. Rev. Finan. Stud. To appear.

Reiss, R.-D. and Thomas, M. (1997). Statistical Analysis of Extreme Values. Birkhäuser, Basel, Switzeland.

Richardson, M. and Smith, T. (1993). A test for multivariate normality in stock returns. $J$. Bus. 66, 295-321

Rockinger, M. and Jondeau, E. (2002). Entropy densities with an application to autoregressive conditional skewness and kurtosis. J. Econometrics 106, 119-142.

Schlather, M. (2001). Examples for the coefficient of tail dependence and the domain of attraction of a bivariate extreme value distribution. Statist. Probab. Lett. 53, 325-329.

Sibuya, M. (1960). Bivariate extreme statistics I. Ann. Inst. Statist. Math. 11, 195-210.

Smith, R. L. (1987). Estimating tails of probability distributions. Ann. Statist. 15, 1174-1207.

Smith, R. L. (1989). Extreme value analysis of environmental time series: an approach to trend detection in ground-level ozone. Statist. Sci. 4, 367-393.

Smith, R. L. and Weissman, I. (1994). Estimating the extremal index. J. Roy. Statist. Soc. Ser. B 56, 515-528.

Stărică, C. (1999). Multivariate extremes for models with constant conditional correlations. $J$. Empirical Finance 6, 515-553.

Tawn, J. A. (1988). Bivariate extreme value theory: models and estimation. Biometrika 75, 397-415.

Zakoïan, J. M. (1994). Threshold heteroskedastic models. J. Econom. Dynam. Control 18, 931-955.

Manchester School of Accounting and Finance, University of Manchester, Oxford Road, Manchester M13 9PL, U.K.

Email: ser-huang.poon@man.ac.uk

HEC-BFSH 1, Department of Management, University of Lausanne, CH-1015 Dorigny, Switzerland.

Email: michael.rockinger@hec.unil.ch

Department of Mathematics and Statistics, Lancaster University, Lancaster, LA1 4YF, U.K.

Email: j.tawn@lancaster.ac.uk

(Received May 2001; accepted July 2003) 